\title{
Bauman e Habermas su teoria e prassi. Alle origini di un confronto incompiuto di Luca Corchia*
}

\section{Riassunto}

Dopo aver constatato l'inesistenza di un reciproco confronto, lo scopo della ricerca si è ridefinito nel ricostruire l'influenza degli scritti di Habermas sull'opera di Zygmunt Bauman - un aspetto noto agli studiosi del sociologo polacco ma poco conosciuto nella comunità sociologica internazionale. Seguendo un approccio filologico e critico letterario, le interpretazioni baumaniane - selettive e discontinue - sono state sistematizzate in due principali temi: 1) i fondamenti conoscitivi della teoria sociale; 2) i fondamenti normativi della teoria critica (e il rapporto con la prassi).

Parole chiave: Bauman; Habermas; sociologia; (post)modernità; prassi.

\begin{abstract} relationship with praxis).

* Università di Pisa. luca.corchia@sp.unipi.it
\end{abstract}

After noting the absence of a mutual confrontation, the aim of this research has been redefined in reconstructing the influence of Habermas' writings on the work of Zygmunt Bauman - an aspect known to scholars of the Polish sociologist but not very well recognized in the international sociological community. Following a philological and critical literary approach, the Baumanian interpretations - selective and discontinuous - have been systematized into two main topics: 1) the epistemological foundations of social theory; 2) the normative foundations of critical theory (and the

Keywords: Bauman; Habermas; sociology; (post)modernity; praxis.

Sicurezza e scienze sociali VI, 1/2018, ISSN 2283-8740, ISSNe 2283-7523 DOI: 10.3280/SISS2018-001012 


\section{Introduzione}

Editorialmente attivi dai primi anni Cinquanta dello scorso secolo e quasi coetanei, Jürgen Habermas e Zygmunt Bauman sono, senza dubbio, tra $\mathrm{i}$ sociologi più famosi e influenti nel campo scientifico e nella sfera pubblica. La celebrità dello studioso tedesco, dapprima legata a quella dell'Istituto per la ricerca sociale di Francoforte e alla figura di Adorno - di cui fu giovane collaboratore e assistente, dal 1956 al 1958, e sostenitore nel Positivismusstreit, tra il 1961 e il 1965 -, si è poi propagata grazie la recezione di un programma di teoria della conoscenza e teoria della società con cui Habermas è divenuto uno tra i maggiori filosofi e scienziati sociali del secondo Novecento. In dialogo con il gotha accademico, si è imposto nella sfera pubblica con interventi sui problemi del nostro tempo (Cfr. Müller, Doohm, 2014). Il successo del sociologo polacco, anch'egli oltre gli ambienti universitari, si deve agli studi - accolti bene dalla comunità internazionale sul socialismo, classi sociali ed ermeneutica, dagli esordi del periodo di Varsavia sino all'approdo a Leeds. La sua fama, però, si è costruita con la vasta produzione che, dagli anni Ottanta, si configura sulla coppia opposizionale "modernità" e "postmodernità" e, infine, sulla metafora della "liquidità" che troverà molteplici declinazioni tematiche (Cfr. Tester, 2004).

In questo saggio ricercheremo le intersezioni fra la teoria critica di Habermas e la "sociologia umanistica" di Bauman, la quale - scriveva Carmen Leccardi - «guarda ai valori umani fondamentali - autonomia, dignità, libertà, giustizia - e alla possibilità della loro espressione senza supporti trascendenti» (2008a: 3). Anche da tale prospettiva intellettuale esamineremo gli aspetti di un confronto, sinora quasi del tutto assente nella letteratura critica, con poche eccezioni di rilievo (Caccamo De Luca, 1982; Wolff, 1983; Beilharz, 2000; Smith, 1999; Bovone, 2002; Giacomantonio, 2014; Almeida, 2015) che non si limitano ai commenti sporadici, di cui terremo comunque conto. La ricerca delle fonti ha mostrato una prima sorpresa: un singolare divario tra l'enorme diffusione editoriale di Bauman e la scarsità di studi monografici - una prova a sostegno dell'immagine che lo raffigura come un outsider nella comunità sociologica (Smith, 1998) e un ulteriore elemento di riflessione. Tra i pochi segnaliamo quelli di Beilharz (2001, 2011), Best (2013), Blackshaw (2005; 2016, a cura di), Chardel (2013), Davis (2008, 2013, a cura di), Davis e Tester (2010, a cura di), Elliott (2007, a cura di), Hviid Jacobsen (2016, a cura di), Hviid Jacobsen e Poder (2008, a cura di), Junge (2006), Kilminster e Varcoe (1995, a cura di), Rattansi (2017), Tester (2004; trad. it. 2005), Tester e Hviid Jacobsen (2005) e Tester, Hviid Jacobsen e Marshman (2007). Per la recezione italiana, anco- 
ra acerba, si possono consultare i testi di Bordoni (2016, a cura di), Capuano (2006), Cipriani (2013), Conte (2011), Giaccardi e Magatti (2001), Leccardi (2008a; 2008b), Magatti (2003), Palese (2012) e Picchio (2012).

Se osserviamo, invece, i riferimenti reciproci interni alle opere dei due autori sorprende l'assoluto disinteresse di Habermas, il quale non si è certo distinto per la chiusura di orizzonti tematici e disciplinari e il cui approccio ricostruttivo lo ha condotto a consultare, interpretare, sistematizzare e appropriarsi di una quantità impressionante di risultati prodotti da una vasta comunità scientifica. Risulta considerevole, per contro, l'attenzione su Habermas da parte di Bauman, come ebbe a rimarcare Dennis Smith: «Nei primi decenni, fino ai primi anni '80, Bauman era soprattutto influenzato da tre scrittori: Karl Marx, Antonio Gramsci e Jürgen Habermas» (1999: 27).

La presenza dello studioso tedesco nel suo orizzonte culturale è testimoniata dalla recensione che Bauman scrisse al testo The Politics of Social Theory: Habermas, Freud and the Critique of Positivism (1981) di Russell Keat. Nell'incipit leggiamo che «L'Habermasologia è una delle pochissime industrie britanniche apparentemente non colpite dalla depressione. Un numero sempre crescente di scrittori si è posto il duplice compito di "scoprire cosa ha realmente detto Habermas" e "correggere gli errori di Habermas" [...] Habermas è stato recentemente corretto in questo paese così tante volte e in tanti modi, che le correzioni stesse formeranno presto un oggetto sufficientemente voluminoso per un prossimo campo di indagine nei dottorati di ricerca» (1983a: 154). Bauman avvertiva che in questi esercizi interpretativi, alla fine, ciascuno finisce per trovare l'Habermas che cerca, tra i filosofi, come Keat, e tra i sociologi, con inevitabili letture distorsive.

Mi interessa dimostrare che valutazioni analoghe possono essere espresse sulla sua lettura di Habermas: selettiva, orientata dal proprio percorso di ricerca, fortemente condizionata dalla prima recezione, quella degli anni di formazione, gradatamente calante e non priva di fraintendimenti - aspetti che ricorrono sovente nella conoscenza reciproca tra grandi autori. L'intervista in appendice al volume Intimations of Postmodernity è un buon esempio. Richard Kilminster e Ian Varcoe gli chiedevano conto delle similitudini tra la sua sociologia e la teoria critica della Scuola di Francoforte e, in particolare, del suo "convinto sostegno" al lavoro di Habermas. Bauman confermava l'adesione alla "sociologia critica", dichiarando, tuttavia, che Habermas "non gli piaceva". Poi, invitato a esplicitare il giudizio, precisava i motivi per cui "non gli piaceva più": «Penso che ciò che mi ha attratto di Habermas, in realtà, è stato il suo ideale di una società strutturata sul modello di un seminario di sociologia, cioè che vi sono solo partecipanti e l'unica cosa che conta è il potere comunicativo, per cui la funzione della 
sociologia è di screditare gli altri fattori, che si nascondono, presumibilmente, dietro l'apparente libera discussione ed eliminarne l'influenza. Una volta raggiunto l'obiettivo, il problema della verità si fonde con quello del consenso [...] Io avevo apprezzato il focus su questo immaginario utopico. Un po' come l'idea dell'esperimento ideale, che naturalmente non è mai raggiunto ma sempre presupposto, altrimenti neppure si sperimenterebbe. Mi era piaciuto questo orizzonte [...] Ma, una volta che Habermas si è trasformato in un semplice rifacimento positivistico di Parsons, ho perso ogni affinità spirituale con il suo progetto» (1992c: 217). La discussione termina qui e non viene chiarito il senso di una critica che potrebbe trovare riscontri in una teoria dell'evoluzione dall'impianto funzionalistico, pur con "importanti revisioni" - si legge nella Theorie des kommunikativen Handelns (1981a) - poco citata da Bauman. L'accostamento viene compreso se si considera un passaggio dell'intervista rilasciata a "Telos", lo stesso anno: «L'ultimo grande tentativo di presentare il mondo come un sistema ordinato fu la teoria di Talcott Parsons: un tentativo d'imporre su un mondo disorganizzato un certo tipo di ordine, scegliendo alcuni aspetti come normali ed accantonandone altri come anormali, devianti, trasgressivi, ecc.» (1992b; trad. it. 2005: 36). In tale direzione si muove Beilharz, rintracciando una delle ragioni della presa di distanza da Habermas nel progressivo rigetto del problema dei fondamenti normativi della società che pone lo studioso tedesco in continuità rispetto a Durkheim e Parsons: «Bauman si interessa sempre meno a Habermas, man mano che Habermas si avvicina al labirinto Durksoniano» (1990: 42). Ciò che distanziava i due studiosi era la questione dei fondamenti del sapere sociologico, che per Habermas coincide con la condizione di possibilità della teoria critica - il punto di vista normativo che controfattualmente rileva il patologico. Per contro, Bauman sembra considerare il problema come sintomo dell'impulso moderno al disciplinamento cognitivo e comportamentale. Infine, se il programma habermasiano è esplicitamente volto alla costruzione di una teoria generale della società, Bauman sembra rifiutarne l'intento, come rilevarono comparativamente sia Beilharz (2000: vii) - «Il lavoro di Bauman è ampio, impressionante, difforme; nega la natura sistemica o la traiettoria relativamente chiara che caratterizza altri corpi di lavoro come, per esempio, quelli di Habermas» - che Keith Tester e Michael Hviid Jacobsen - «Bauman non ha scritto una teoria sociologica coerente, integrata o sistematica» (2005: 20). Sul giudizio vi è piena sintonia tra gli studiosi, come conferma Tony Blackshaw, citando un brano di Michael Pusey (1987: 26): «Si deve comprendere sin dall'inizio che lo scopo della sociologia di Bauman non è quello di ricostruire "i testi classici basilari della sociologia come elementi 
fondanti di una teoria generale della società", alla maniera di Habermas» (2005: 16). Carmen Leccardi ha precisato che la scarsa sistematicità - motivo di frequenti critiche nella comunità scientifica - riguarda tanto la «voluta lontananza da un'architettura accademica tradizionale» quanto «la sua scelta di procedere nell'esplorazione del mondo sociale per frammenti, in apparenza senza una direzione precisa, con molti riferimenti teorici e molte ispirazioni, ma pochi o pochissimi principi metodologici» (2008a: 33).

Nelle pagine seguenti ricostruiremo la recezione baumaniana dell'opera di Habermas, dagli inizi, fortemente elogiativi, sino al distacco e ai giudizi tanto più sferzanti quanto più problematici. La svolta è avvenuta a partire dalla trilogia Legislators and Interpreters, Modernity and the Holocaust e Modernity and Ambivalence, scritti da Bauman tra il 1987 e il 1991. Come banco di prova, ci concentriamo su due aspetti centrali nella riflessione dell'epoca: i fondamenti della teoria sociale (2) e il nesso tra teoria sociale e prassi (4), con un intermezzo sulla modernità (e sulla postmodernità) (3).

\section{I fondamenti conoscitivi della teoria sociale}

Dai primi anni Sessanta alla fine degli anni Ottanta, la produzione editoriale di Bauman concerne la letteratura sociologica internazionale, la riflessione metodologica sull'approccio ermeneutico e la rielaborazione dei concetti fondamentali: cultura, strutture sociali, élite, classi, capitalismo, lavoro, consumo, democrazia, socialismo, totalitarismo, ideologia, partiti politici, media, ecc. Il progetto scientifico era quello di riattualizzare il marxismo come quadro di riferimento teorico e modello dei rapporti tra teoria e prassi, mentre la finalità pratica era promuovere l'emancipazione dell'umanità. Nell'elaborazione di questo complesso programma epistemologico, teoretico e pratico, Bauman scopre relativamente presto gli scritti di Habermas.

In chiusura del volume Culture as Praxis (1973), tradotto subito e recensito anche in Italia (Penati, 1977), Bauman manifestava apprezzamento verso il progetto di ricerca che Habermas aveva inaugurato nella prolusione del 1965 e concluso con Erkenntnis und Interesse (1968; trad. it. 1970), noto al sociologo polacco nella traduzione della Beacon Press del 1971. L'attenzione di Habermas era rivolta alle categorie del lavoro e del linguaggio, al fine di ricostruire i fondamenti dell'azione e dell'intersoggettività e i presupposti antropologici delle forme di sapere metodicamente strutturate, oltre a cercare le radici dell' "interesse emancipativo" nella filogenesi umana. L'aspetto che interessava Bauman era il collegamento della riflessione metodologica sulle condizioni e limiti conoscitivi delle scienze analitiche 
ed ermeneutiche con la ricostruzione delle forme di esperienza, cioè il «tessuto vero e proprio del processo vitale umano» (1973; trad. it. 1976: 250).

In quegli anni, il confronto con Habermas equivaleva alla scoperta di uno dei progetti più interessanti di rinnovamento della teoria critica. La collocazione di Bauman nel solco di questa tradizione era confermata da David Held (1978), in una recensione di Towards a Critical Sociology. An Essay on Commonsense and Emancipation (1976b; trad. it. 1982). Lo studioso polacco evidenziava le posizioni eterogenee nella Scuola di Francoforte, la quale non costituiva più un paradigma unitario, se mai lo fosse stato. Viceversa, Held individuava due modelli, peraltro disomogenei al loro interno e variamente rielaborati nel tempo: quello dei fondatori (Horkheimer, Adorno e Marcuse), e quello della seconda generazione, in cui Habermas assumeva una posizione dominante. Il testo di Bauman veniva, quindi, presentato positivamente per l'originalità argomentativa, inusuale nell'accademia inglese, e l'apertura alla fenomenologia, all'ermeneutica e alla teoria critica, che egli contrapponeva, per un verso, al marxismo ortodosso, per altro verso, agli approcci convenzionali nelle scienze sociali. Con la tradizione critica, egli condivideva l'interesse verso le condizioni di un'indagine interdisciplinare su alcune questioni cruciali, quali il rapporto tra individuo e società, il significato della cultura, gli elementi che rendono possibile la riproduzione e trasformazione della società, oltre alla riflessione epistemologica sui fondamenti della conoscenza sociale e all'idea del sapere sociologico come critica dell'ideologia e prassi intellettuale volta a generare processi riflessivi di autochiarimento nella sfera pubblica (Ivi, 554). Il richiamo sempre più esplicito all'opera di Habermas era dovuto alla medesima esigenza conoscitiva, teorica e pratica, come aveva indicato con precisione Rita Caccamo De Luca nell'introdurre la traduzione italiana: «elaborare una teoria della società che parta da una critica della conoscenza nel suo sviluppo storico e nella formazione delle sue categorie costitutive per giungere a fondare un nuovo rapporto tra sapere e prassi» (1982: 18).

Questo è il progetto che Bauman elaborò in Towards a Critical Sociology, facendo riferimento alla raccolta di saggi Theorie und Praxis (1963) dedicato da Habermas al dualismo tra conoscenza tecnica e pratica - quale pietra angolare per interpretare lo sviluppo della società moderna - e tradotto parzialmente dalla Beacon Press, tre anni prima. Bauman definiva i termini di una sociologia critica che non "naturalizza" la società, come invece fa il positivismo, riordina la conoscenza esperienziale in termini di strutture storiche e rivela le potenzialità umane non compiute (1976b; trad. it. 1982: 142). Egli citava, inoltre, alcuni brani dell'Introduzione alla seconda edizione, il cui Habermas coglieva «una buona occasione per verificare attra- 
verso una retrospettiva (necessariamente rapida) dove mi hanno portato le riflessioni fatte finora» (1971a; trad. it. 1973: 29). In particolare, la parte esaminata da Bauman era quella riservata alle "indagini storiche" e "riflessioni esplorative" del libro Conoscenza e interesse, il cui intento era delineare «il programma di una teoria della scienza che deve cogliere sistematicamente il contesto di costituzione e di utilizzazione delle teorie scientifiche in generale» e che, al tal fine, poneva la «questione dei sistemi dei concetti fondamentali (o del "quadro trascendentale"), entro i quali organizziamo le nostre esperienze a priori e prima di ogni scienza» (Ivi, 37). La questione riguardava il fondamento conoscitivo della teoria sociale, la prima grande direttrice degli scritti di Habermas. L'esposizione di Bauman della sintesi habermasiana ripercorre a grandi linee la questione metodologica del dualismo tra "scienze empirico-analitiche, fondate sull' esperienza dell'"agire strumentale", e "scienze ermeneutiche", radicate, per contro, nella prassi delle "interazioni linguistiche". Egli descriveva in modo compiuto il problema e la soluzione proposta: «Per quanto la comunicazione possa essere considerata una condizione antropologica, generica dell'uomo, gli interessi tecnici e pratici emergono immediatamente da ogni tipo di comunicazione, come tentativi inevitabili per "chiarire la costituzione dei fatti sui quali sono possibili affermazioni teoriche"» (1976b; trad. it. 1982: 188).

Bauman condivideva l'idea che la comprensione e l'intesa siano già attivi pre-riflessivamente nell'"agire comunicativo" del mondo della vita e, su questa base, trovino il proprio fondamento le scienze che formalizzano la "logica di domanda e risposta" del linguaggio quotidiano nel "discorso". Ciò che maggiormente lo interessava, quindi, era la sezione Azione e discorso, in cui Habermas introduceva un primo abbozzo della differenziazione tra le "pretese di validità" - comprensibilità delle espressioni, verità delle proposizioni, giustezza delle norme-adeguatezza dei valori e veridicità delle intenzioni - accettate ingenuamente nelle azioni e, se problematizzate, dimostrate o meno valide nelle argomentazioni. Gli interessi conoscitivi tecnici e pratici condividono la condizione che: «Dalla "comunicazione" l'articolazione pre-riflessiva della pratica abituale, il riconoscimento di senso comune dei "fatti" - essi staccano il "discorso", libero dalle costrizioni immediate dell'azione, che è soggetto a regole sue proprie, ragionate, ed è in grado di fornire una giustificazione ragionata di ciò che è stato semplicemente riconosciuto come fattuale» (1976b; trad. it. 1982: 187-188).

Bauman esaminava, poi, la replica con cui Habermas, nella sezione $O g$ gettività della conoscenza e interesse, aveva inteso rispondere all'obiezione sull'insufficiente chiarimento dello statuto degli "interessi-guida", ancorando, per così dire, le giustificazioni argomentative di discorsi scientifici 
svincolati dalla coazione all'azione, alle pratiche comunicative dell'agire quotidiano - tanto nelle scienze empirico-analitiche quanto in quelle ermeneutiche: «È grazie alla relativa autonomia del discorso che le affermazioni teoretiche sul dominio fenomenico delle cose e degli accadimenti (nel caso dell'interesse tecnico), o di persone e di articolazioni del pensiero (nel caso dell'interesse pratico), si possono fare e giustificare. L'autonomia del discorso non è mai completa. È sempre messa in moto dalle necessità o dagli interrogativi che sorgono dall'interno della pratica di comunicazione; e ci si aspetta che i suoi risultati, se sono di applicazione pratica, abbiano effetto retroattivo nella corrente principale dell'azione orientata e degli orientamenti della comunicazione quotidiana» (Ivi: 188). Se la costituzione degli ambiti oggettuali delle scienze empiriche può essere intesa come la prosecuzione delle "oggettivazioni" che operano nel mondo della vita prima di ogni scienza, per altro verso, la pretesa di oggettività presuppone «una virtualizzazione della pressione dell'esperienza e della decisione, che sola ci consente un esame discorsivo di pretese ipotetiche di validità e, in tal modo, la generazione di un sapere fondato» (1971a; trad. it. 1973: 52). Il processo conoscitivo con cui si giustificano le asserzioni teoriche - si trasforma il "meramente riconosciuto" nell" "effettivamente conosciuto" - è racchiuso nel discorso, in cui è intenzionalmente impostato e controllato metodologicamente dal proprio sistema di regole (1976b; trad. it. 1982: 188).

Il punto culminante dell'adesione alla concezione habermasiana di teoria sociale fu la pubblicazione di Hermeneutics and Social Science, in cui si affermava che «il modello di accordo razionale di Habermas [...] avvicina la metodologia della scienza comprendente all'ideale della critica guidata dalla verità, così come le regole della pratica sperimentale vi hanno condotto le scienze empirico-pratiche» (1978: 243-244; cfr. Smith, 1999: 177).

Nei primi anni Ottanta, Kurt H. Wolff rilevava l'interesse di Bauman per la linea trascendentale della filosofia critica tedesca, da Kant a Husserl, a cui la sociologia deve l'interesse epistemologico per gli a-priori della relazione soggetto-mondo, sul piano della conoscenza e dell'esperienza pratica. L'ex Presidente della Società Internazionale per la Sociologia della Conoscenza confermava l'interpretazione dell'approccio metodologico di Bauman sulla lettura di un manoscritto che sarà pubblicato, solo un decennio dopo con il titolo Teoria critica (1991b; trad. it. 2005, 164-188). Nel testo sono frequenti i riferimenti a Habermas, compreso ancora nel quadro del programma di ricerca culminato in Erkenntnis und Interesse (1968), in cui era proposto "un modo di teorizzare" riflessivo che problematizza i presupposti conoscitivi del sapere sociologico e, quindi, richiede la «collocazione della teoria al centro dell'analisi, 
rifiutando di accettarne l'autorità senza alcuna prova, esigendo che le ragioni sulla base delle quali questa autorità viene proclamata vengano svelate, e, infine, ponendo in discussione queste ragioni [...] Vi sono molti inespressi presupposti della conoscenza che la teoria critica costringe a venire allo scoperto e formula come argomenti d'analisi, sospendendo l'accettazione di qualsiasi teoria sia basata su di essi fino a quando questi presupposti siano messi chiaramente in luce» (1991b, trad. it. 2005, 164165). Bauman attribuiva alla "seconda generazione" (Habermas e Karl Otto Apel), il merito di aver ricostruito il "ponte" che collega la teoria critica alla tradizione epistemologica che assume il compito di dotare la conoscenza scientifica di un "metodo per verificare la verità" delle asserzioni - un nesso - egli scrive - «eliminato dalla disincantata generazione precedente in maniera fin troppo sconsiderata» (Ivi: 179). Inoltre, nel saggio era compendiata la teoria sullo "statuto quasi-trascendentale" degli interessi conoscitivi: "Jürgen Habermas definì gli interessi che sono alla base delle due categorie di conoscenza come trascendenti-la-specie (species-transcendental). Sono trascendenti, poiché senza di essi non è possibile una vita sociale; essi sono, per così dire, condizioni previe di tutte le forme d'esistenza umana. Sono, però, trascendenti la specie, poiché la loro ubiquità può essere fatta risalire alla forma caratteristica che la specie umana raggiunse nel corso dell'evoluzione; mano e lingua, lavoro e linguaggio, si sono sviluppati tutti come gli strumenti principali di questa sopravvivenza e perfettibilità della specie. Essendo presenti dovunque nel modo specificamente umano di vita, gli interessi per la padronanza (interesse tecnico) e per la cooperazione (interesse pratico) determinano il modo in cui l'esperienza umana viene collettivamente percepita, categorizzata e tipizzata. Gli aspetti dell'esperienza sono scelti secondo la loro attinenza ai due interessi fondamentali. Questo è ciò che tutti noi, come singoli individui e come comunità, facciamo continuamente» (1991b; trad. it. 2005: 182).

Bauman conosceva, allora, oltre a Erkenntnis und Interesse, la traduzione di Legitimationsprobleme im Spätkapitalismus, data alle stampe nel 1976, mentre ignorava Zur Rekonstruktion des Historischen Materialismus, tradotto nel 1979, e gli scritti del periodo di Starnberg (1971-1981), molti pubblicati in inglese da riviste internazionali, in cui stava maturando l'approccio ricostruttivo di Theorie des kommunikativen Handelns (1981a). È rilevante perché Bauman non si accorge del mutamento paradigmatico verso le scienze ricostruttive che trova applicazione nell'analisi della logica di sviluppo ontogenetico e filogenetico (Corchia, 2007a, 2009, 2010). In 
ogni modo, nel corso del decennio, Bauman muterà radicalmente posizione non appena prenderà corpo l'antinomia tra modernità e postmodernità.

In Legislators and interpreters: On Modernity, Post-Modernity, Intellectuals - un testo recensito, tra gli altri, da Lewis A. Coser (1989) -, il sociologo della postmodernità condivide la ricostruzione habermasiana del processo di razionalizzazione culturale nei due aspetti della riflessività della conoscenza e della differenziazione delle forme specialistiche del sapere (scienza, morale ed estetica) dalle visioni metafisiche e religiose del mondo per le questioni di verità, giustizia e gusto. Un brano analogo di Modernity aggiunge la giusta collocazione di quell'analisi all'interno del quadro di riferimento delle tre critiche kantiane, con la «divisione tra ragione pura, ragione pratica e giudizio» (1993; trad. it. 2005: 195). Habermas avrebbe ignorato, tuttavia, un secondo tipo di pluralismo, relativo alla pluralizzazione di discorsi intessuti di elementi comuni e tradizionali e il cui riconoscimento - sostanzialmente nuovo - «è difficilmente riconciliabile con lo spirito e la pratica della modernità. In certo modo, riunendo i due tipi di pluralismo, Habermas preclude la possibilità di considerare l'attuale situazione dell'intellettuale occidentale come sostanzialmente nuova; e richiede mutamenti piuttosto estesi nel modo in cui i servizi intellettuali sono stati tradizionalmente dispensati» (1987; trad. it. 1992: 148). Le considerazioni di Bauman scaturiscono solo da un confronto con la teoria dell'evoluzione sociale, qui desumibile dalla lettura del saggio Questions and Counterquestions (1984), in cui Habermas rispondeva ai commenti e alle critiche raccolte da Richard J. Bernstein nel volume Habermas and Modernity (1985).

Il senso della critica di Bauman sarà precisato nella recensione della traduzione inglese di Der philosophische Diskurs der Moderne (1985). Dopo aver ripercorso il progetto di fondazione quasi trascendentale degli interessi conoscitivi, Bauman pone Habermas di fronte alla sfida di un pensiero post-moderno - la "nuova eresia" - «che mette in discussione sia la necessità che la fattibilità della certezza (o del consenso comunicativo, del resto) e nega che nella scienza vi sia altro dalle sue radici sociali» (1988b: 473). Il più "fermo" e "persuasivo" difensore del discorso moderno, tuttavia, non avrebbe distinto tra i due aspetti della postmodernità, la "strategia" e la "diagnosi", che necessariamente non vanno considerati e confutati insieme.

La strategia postmodernista riguarda la rinuncia alla fondazione universale della conoscenza a favore di una riconciliazione con la natura intrinsecamente particolare e relativa di ogni esperienza umana: «secondo tale strategia, la conoscenza acquisisce e mantiene il proprio senso solo all'interno della sua forma di vita "nativa", delle tradizioni comunitarie, delle comunità linguistiche, ecc; ciò che rimane è unicamente il compito di traduzione e 
interpretazione da svolgere nello spazio condiviso» (Ivi: 474). Contro il postmodernismo come strategia, Habermas avrebbe impiegato tutte le proprie armi argomentative, sebbene Bauman ritenga che in esse siano rintracciabili elementi pronti per essere "trapiantati" nell'impostazione postmodernista, in particolare la teoria consensuale della verità e la critica dell'avalutatività scientifica. In tal senso, si legge che «per quanto fortemente Habermas insista sull'affiliazione modernista della sua teoria della verità e della conoscenza, c'è ben poco nella teoria stessa (tranne il modo in cui Habermas la rivitalizza nelle fondamenta della storia intellettuale moderna) che può renderla immune a tali appropriazioni postmoderniste» (Ibidem).

Va precisato che, per Bauman, se la postmodernità in quanto diagnosi è un'ipotesi valida, difficilmente la strategia postmoderna contribuirà a farcela comprendere: «Abbiamo bisogno che le scienze sociali moderniste comprendano correttamente e teorizzino correttamente la società contemporanea come postmodernità. In altre parole, abbiamo bisogno di una sociologia della postmodernità e non di una sociologia postmoderna» (Ibidem). Sul piano metodologico, l'approccio baumaniano continua a favorire una sociologia ermeneutica, che egli riteneva di aver compiutamente elaborato negli scritti Hermeneutics and Social Science: Approaches to Understanding (1978) e Hermeneutics and Modern Social Theory (1989b) e impronta il suo particolare stile narrativo e conversazionale (cfr. Dawson, 2015). Quest'ultimo è un aspetto tutt'altro che secondario nell'autocompresione di Bauman. Come sottolinea Carmen Leccardi, la sua produzione è «sempre attenta, in modo creativo e sull'onda di un'immaginazione e uno stile espositivo per più di un verso letterari, a recuperare la relazione tra aspetti micro e macro-sociali, tra vissuti personali e vita politica e sociale» (2008a: 3). Così "eclettica" in estensione e intenzionalmente "narrativa", essa ricerca l'immediata eco al fine di meglio caratterizzarsi come una sociologia "impegnata" nella sfera pubblica, capace di incidere nello spirito del tempo. Più sofisticata, invece, risulta la prosa dello studioso tedesco, anche negli interventi giornalistici comunque seguiti da cerchie di lettori e dai politici.

Tornando al nucleo della critica di Bauman - l'incapacità habermasiana di comprendere il secondo tipo di pluralismo -, egli manifesta, non senza enfasi, la convinzione che non sia possibile alcuna fondazione metafisica $o$ trascendentale del sapere sociologico che ci sollevi, volta per volta e contesto per contesto, dalla ricerca discorsiva della comprensione e dell'intesa. In un brano di Philosophical Affinities of Postmodern Sociology, questa posizione era così caratterizzata: «Data la nostra conoscenza della contingenza - che ora si estende dall'idea del bello a quella dell'essere stesso, alla sua verità e alla sua ragione - non possiamo abbandonare la ricerca del 
consenso: sappiamo dopo tutto che l'accordo non è predeterminato e non è garantito in anticipo, che non può basarsi su nient'altro che la nostra argomentazione. Il nostro è il coraggio della disperazione. Non possiamo che raddoppiare i nostri sforzi passando di sconfitta in sconfitta» (1990b: 138). Nuovamente, Bauman oppone la consapevolezza della fallibilità del pensiero sociale al modello habermasiano di "comunicazione non distorta", descritto come «una prospettiva realistica consensuale sulla verità» (Ibidem). Ancor più recisa sarà la risposta nell'intervista di Emanuele Caroppo e Patrizia Brogna, in cui, ribadendo la convinzione del "dover imparare a vivere temporaneamente con le differenze", egli affermava: «Non sono d'accordo con Habermas, che parla di un ideale di comunicazione in cui alla fine della storia c'è il consenso e tutti sono d'accordo. Secondo me questo non è un obiettivo auspicabile, perché se tutti la pensassimo allo stesso modo basterebbe una persona sola e gli altri sarebbero dei cloni» (2008: 162).

L'interpretazione è sorprendente se si considera che, in Towards a Critical Sociology (1976b), egli aveva puntualmente commentato il saggio in cui Habermas presentava la logica della "comunicazione linguistica non distorta", introdotta in Vorbereitende Bemerkungen zu einer Theorie der kommunikativen Kompetenz (1971b) - rielaborazione del seminario di sociologia del corso universitario del 1969-70 dedicato ai Probleme der Sprachsoziologie - e condensata nell'Introduzione del 1971.

In ogni modo, Bauman ripresenterà letteralmente tale critica in Modernity and Ambivalence (1991a; trad. it. 2010: 250), in cui viene descritto anche il paradosso di una ricerca delle basi consensuali che porta a maggiore frammentazione: «La spinta alla sintesi è il fattore principale delle biforcazioni senza fine. Ogni tentativo di convergenza e sintesi porta a nuove fratture e divisioni. Quella che si presume essere la formula per un accordo che ponga fine a ogni disaccordo dimostra di essere, nel momento in cui è stata formulata, un'occasione di nuovo disaccordo e di nuove pressioni a ritrattare. Tutti gli sforzi per solidificare le strutture sciolte del mondo della vita provocano una maggiore fragilità e tendenza a dividersi» (Ivi: 251). Per Bauman, l'unico consenso che potrebbe avere una possibilità di successo, in termini universali, sarebbe l'accettazione dell'eterogeneità dei dissensi. Neppure dovremmo sperare ormai che basi solide possano essere costruite.

La condizione postmoderna è quella delle antinomie e dissociazioni, tra possibilità che si dischiudono e minacce nascoste dietro ogni opportunità. Anche l'espressione della modernità come "progetto incompiuto" dovrebbe essere intesa come presa di coscienza della natura ambivalente delle cose e dell'impossibilità di appagare il desiderio di certezze: «Il fatto stesso che si parli ora di modernità come di un progetto (un progetto con intenzioni, fini 
e mezzi) testimonia in modo più convincente il cambiamento che è avvenuto in noi. I nostri antenati non hanno parlato del "progetto" quando erano attivamente impegnati in quello che ora ci sembra un affare incompiuto». (Ivi: 271). Oramai, Bauman ha abbandonato definitivamente Habermas e le sempre più sporadiche affermazioni critiche sono esemplificate da Life in Fragments, allorché Bauman così condensava il proprio pensiero: «La voce della coscienza, che è la voce della responsabilità, è percepibile sono nella discordanza dei suoi dissonanti. Il consenso e l'unanimità preannunciano la tranquillità del cimitero (la "comunicazione perfetta" di Habermas, che misura la propria perfezione sulla base del consenso e dell'eliminazione del dissenso, è un altro sogno mortale che propone una cura definitiva ai mali della libertà); nel cimitero del consenso universale, la responsabilità, la libertà e l'individuo esalano il loro ultimo respiro» (1995; trad. it. 1999: 5).

Oltre alla discutibile interpretazione del significato della situazione linguistica ideale, l'analisi di Bauman palesa un fraintendimento riguardo alla funzione di questa figura habermasiana. L'intento della critica si palesa in un'intervista di Harald Welzer, nel 2002, in cui si dichiara che la lettura degli scritti di Habermas «era una perdita di tempo poiché contenevano un «mondo popolato da concetti e non da persone [...] feticismo teorico [...] separato dal mondo reale». Il brano è riportato da Larry Ray, il quale commentava che si tratta di «una critica a cui molta sociologia, compresa quella di Bauman, potrebbe essere soggetta» (2007: 68) Il distacco del sociologo della liquidità non solo dall'autore della Teoria dell'agire comunicativo bensì dalla teoria in quanto tale (e dalla ricerca empirica), a favore di una costruzione concettuale metaforica, è inteso come «sintomatico della direzione presa dalla sociologia negli ultimi vent'anni [...] in cui le teorie sono diventate metafore che descrivono l'esperienza umana, con tentativi generalizzati di cogliere il significato della vita contemporanea» (Ibidem). Felipe Quintão Almeida, in un recente saggio sulla parabola discendente del giudizio su Habermas, presenta una descrizione plausibile di altri elementi che indussero Bauman a colorire con tonalità emotive ostili - una vera e propria "antipatia" - le critiche al progetto di teoria sociale e al concetto di situazione linguistica ideale: "Valutiamo che questa indisposizione sia associata, per un verso, alla critica habermasiana della postmodernità (e del presunto conservatorismo relativo a tutte le questioni rilevanti) e, per altro verso, alla critica baumaniana dell'“ordine come compito della modernità", per come si sviluppa nei libri che compongono la sua trilogia» (2015: 59).

È con questa cassetta degli attrezzi e tali disposizioni che Bauman colloca l'interpretazione di Habermas nella tenaglia modernità-postmodernità. 


\section{Intermezzo sull'antinomia fra modernità e postmodernità}

Il secondo elemento del discorso postmodernista, per Bauman, infatti, è la diagnosi della società attuale come "qualitativamente distinta" da quella moderna, un «tipo di società che può essere teorizzata, per così dire, come sistema a sé stante, piuttosto che come una forma malata della precedente» (1988b, 474). È questa tesi che rese popolari, molto commentati e criticati, i suoi scritti nella comunità sociologica internazionale (Therborn, 2006: 198). Sotto tale aspetto, emergerebbe il difetto più "sorprendente" dell'autore di Legitimationsprobleme im Spätkapitalismus (1973; trad. it. 1975), che più di altri studiosi aveva analizzato compiutamente le trasformazioni $\mathrm{e}$ crisi delle "società mature". Nei primi anni Settanta, il modello di scambio tra economia e politica, da un lato, e tra i sistemi funzionali e $\mathrm{i}$ processi di riproduzione simbolica del mondo della vita, dall'altro lato, stava dando segnali di disequilibrio che la letteratura sociologica aveva ben colto. Quella di Habermas fu una delle analisi più compiute dei fenomeni patologici, a cui Bauman continua a far riferimento dedicandogli, oltre a una recensione elogiativa (1976c), alcuni brani del saggio Class: Before and After (1982).

Sul finire degli anni Ottanta, tuttavia, i rilievi critici diventano predominanti. In Sociological Responses to Postmodernity, Bauman critica il testo di Habermas - accomunato a quelli di Claus Offe, James O'Connor e André Gorz - per non aver colto che lo stato di crisi della modernità dischiude oramai un "tipo sociale a sé stante" che definisce "postmoderno": "Ciò che manca in queste teorie è il tentativo di considerare le manifestazioni culturali della postmodernità come aspetti di una più ampia, sistemica, trasformazione, sia che si tratti dell'emergere di un nuovo tipo di sistema sociale, sia che si tratti di una "crisi" di quello vecchio [...] Ciò che unisce le loro teorie (così differenti sotto molti aspetti) è l'assunto che il carattere distintivo della società contemporanea, altrove (ma non in queste teorie) diagnosticato come l'avvento della postmodernità, possa essere meglio inteso come una deviazione dal modello ortodosso della società moderna; una deviazione causata dall'attuale incapacità del sistema sociale di assicurare la propria riproduzione nella sua forma antica, "classica"» (1989c: 45). Secondo Bauman, invece, i tempi erano maturi per comprendere che lo sviluppo delle forze economiche stava generando le condizioni per la costituzione di un nuovo principio di organizzazione sociale, discontinuo rispetto alla fase precedente della storia capitalista, e sempre meno dipendente dal sistema statuale e dai legami con i radicamenti territoriali nazionali. La stessa affermazione del pensiero postmoderno sarebbe stata una conseguenza culturale della nuova condizione sociale postmodernista 
(Ibidem), in cui entra in crisi il modo moderno di interpretare il mondo come «un campo governabile con l'ausilio dell'azione razionale» (Leccardi, 2008a: 7). Il concetto di crisi non deve ricordare lo "stato morboso" da cui guarire poiché, per lo studioso polacco, la postmodernità è uno stato vitale.

I due testi più celebri di quel periodo in cui Bauman ha delineato l'opposizione tra modernità e postmodernità sono Modernity and the Holocaust (1989) e Modernity and Ambivalence (1991), a cui possiamo accostare alcuni saggi brevi su tale antinomia. Non ci è possibile procedere nella loro esposizione e rimandiamo agli altri articoli della rivista. Ci limitiamo a poche notazioni che aiutano a comprendere la svolta e la distanza sempre più marcata tra Bauman e Habermas. La prima riguarda l'interpretazione dell'Olocausto come «prodotto specifico dell'incontro tra le vecchie tensioni che la modernità aveva ignorato, trascurato o mancato di risolvere, e i potenti strumenti di azione razionale ed efficiente creati dallo sviluppo della modernità stessa» (1989a; trad. it. 1992: 15). Gli argomenti avanzati nel libro, come noto, destarono molto scalpore nella comunità accademica e nella sfera pubblica, con giudizi più negativi che positivi, nonostante i premi. Non ci risultano negli scritti e nelle cronache commenti di Habermas ma non è difficile immaginare il dissenso, se ne consideriamo tanto la concezione della modernità quanto le analisi storico-sociali dei regimi fascisti.

Il secondo libro significativo di questo periodo è Modernity and Ambivalence (1991a; trad. it. 2010), il cui tema è la fondamentale ambivalenza della modernità occidentale, costretta nella dialettica tra libertà e dominio. Bauman si pone apertamente in linea di continuità con le tesi che Adorno e Horkheimer tratteggiarono nella Dialektik der Aufklärung (1947; trad. it. 1966), nell'accoglimento della duplice tesi weberiana sulla "perdita di senso" e "perdita di libertà", nell'analisi delle tre forme di dominio e nella riduzione della razionalità alla dimensione oggettiva e strumentale. A tale proposito, possiamo dare per note le critiche di Habermas ai "maestri" e supporre, in assenza di fonti dirette, un disaccordo che si estende anche all'analisi della natura del comunismo, alle conseguenze della sua fine e alla persuasione che si stesse aprendo una nuova epoca detta postmodernità.

Per altro verso, dagli anni Novanta, Bauman e Habermas, in maniera indipendente, si interrogano sui fenomeni che le scienze sociali hanno ritenuto cruciali del nostro tempo, avanzando riflessioni, in tanti aspetti, sorprendente simili: l'internazionalizzazione del capitale finanziario e la fine del mondo del lavoro, la crisi della sovranità statuale e il declino del welfare state, i limiti delle soluzioni transnazionali (Ue), l'impoverimento della sfera pubblica, il pluralismo e il métissage culturale, l'erosione delle fonti solidaristiche dei legami, la costruzione di identità nella sfera privata dei con- 
sumi. Molto spesso, inoltre, essi fanno riferimento ai medesimi studi specialistici, in cui cercano conferme parziali alle loro teorie sociologiche. I maggiori punti di convergenza tra $\mathrm{i}$ due studiosi risiedono nella disamina del contesto delle trasformazioni in corso, connotato dalla globalizzazione. Un raffronto sistematico tra le loro concezioni della nuova costellazione su cui è in corso di stesura un saggio con Andrea Borghini - dovrebbe meglio approfondire le distanze incolmabili e gli inattesi punti di contatto. Per il momento, ci interessa mostrare l'altro terreno in cui quell'incontro già è avvenuto, prima segnato dall'intesa, dopo dal dissenso: la concezione del rapporto tra teoria sociale e prassi di vita e dei compiti degli intellettuali.

\section{I fondamenti normativi della teoria critica}

La riflessione su teoria e prassi è costitutiva per due studiosi di formazione marxista che, a lungo, cercarono di rinnovare il materialismo storico e affonda le proprie radici nel contesto intellettuale dei primi anni Settanta.

Nella lettura di Theorie und Praxis (1963; trad. it. 1973), in particolare del saggio Dogmatismus, Vernunft und Entscheidung - Zu Theorie und Praxis in der verwissenschaftlichten Zivilisation, Bauman, in Towards a Critical Sociology, seguiva la critica habermasiana al positivismo non più sul versante epistemologico delle condizioni di conoscibilità dei fenomeni sociali ma su quello pratico del nesso tra teoria sociologica e prassi di vita.

In quel saggio, Habermas muoveva una serie di obiezioni a una concezione positivista della scienza che forniva un ideale regolativo avulso dalla prassi di ricerca sia rispetto al contesto della "scoperta", sia riguardo al contesto della "conferma". Trascurando le condizioni fattuali in cui e per cui agiscono i ricercatori, il positivismo finiva per considerare la scienza come una sorta di "terzo mondo" astorico e asociale assoggettato solo alla logica. Ciò che esula dal rapporto tra proposizioni teoriche e osservative era inaccessibile alla discussione critica e abbandonato alla mera decisione. Dopo aver brevemente riassunto il superamento del riduzionismo positivista del principio di avalutatività che orienta l'autocomprensione scientista, Bauman apprezza la ricostruzione genealogica di quelle credenze e pratiche, riconducendole agli atteggiamenti presenti nella cultura dell'Illuminismo: «Come rilevò Habermas, la possibilità di tale programma era contenuta, sebbene solo in nuce, nella considerazione illuminista della ragione come valore supremo e guida della esperienza umana nel mondo» (1976b; trad. it. 1982: 64). Tralasciando questa rilettura, limitiamoci a rimarcare la coerente adesione di Bauman alla tesi sulla dialettica di un illuminismo che avendo 
affermato "il trionfo della ragione sul pregiudizio", come condizione per l'emancipazione umana, finì per imprigionarla: «I "philosophes" non sapevano e non potevano sapere che il progresso di una conoscenza tecnicamente esperta e strumentalmente efficiente, avrebbe, prima o poi, costretto l'uomo ad un mondo immenso e artificiale dal quale egli sarebbe stato condizionato materialmente ma che non sarebbe dipeso dalla sua capacità di penetrarlo e abbracciarlo spiritualmente» (Ibidem). Nel testo di Bauman, è evidente, risuonano motivi francofortesi, dai quali Habermas si distanzierà: sia dalla critica alla razionalità tecnico-scientifica in quanto tale, sia dalla tesi che la razionalizzazione sia riducibile a tale forma di conoscenza.

Poco dopo, tuttavia, Bauman citava un brano in cui Habermas manifesta una critica al riduzionismo di una scienza orientata al solo agire strumentale che lo accompagnerà in modo permanente, seppur variamente riformulata: «L'irrazionalità della storia si basa sul fatto che noi la "facciamo", senza finora riuscire a farla coscientemente. Una razionalizzazione della storia perciò non può essere promossa da un potere di controllo ampliato di uomini manipolanti, bensì soltanto grazie a un livello più elevato di riflessione, grazie a una coscienza di uomini agenti, che progredisce nell'emancipazione» (1963; trad. it. 1973: 413; Bauman, 1976b; trad. it. 1982: 153). Habermas non nega affatto il ruolo delle scienze empiriche nello studio dei fenomeni socio-culturali. Tuttavia, l'interpretazione positivistica di tali processi è troppo restrittiva. Sul piano conoscitivo, le scienze sociali non mirano solo alla descrizione di stati di fatto, alla scoperta di correlazioni più o meno causali e a previsioni condizionate; così come su quello pratico non sono soddisfatte solo dal successo strumentale delle azioni che ispirano, ad esempio come sapere ausiliario ai fini di un" "amministrazione razionale". Va detto che la consulenza scientifica a favore di imprese e istituzioni pubbliche, in realtà, è costitutiva per la genesi e lo sviluppo delle conoscenze e pratiche, cresce in tutte le società progredite e concorre a tale progresso. Habermas riconosce e apprezza i compiti di "tecnologia sociale", pur avvertendo i rischi di strumentalizzazione delle scienze sociali a fini ideologici. Questa è la chiave di lettura predominante nel pensiero di Bauman, acquisita anche tramite le tesi dell'autore di Theorie und Praxis sulla civiltà scientificizzata e le conseguenze pratiche del progresso tecnico-scientifico.

Tra i compiti tecnico-scientifici, a ben vedere, si collocano pure quelli di una sociologia volta a ricostruire le componenti strutturali e i meccanismi di mutamento delle formazioni sociali e descriverne le patologie. I "classici" del pensiero sociale, con cui Habermas si confronta, intendendo rinnovarne la "lezione", sono animati da questo "spirito sociologico". E lo si ritrova anche in Bauman, secondo il quale "fare sociologia" significa con- 
frontarsi con i grandi problemi del tempo (Beilharz 1990: 42). In Thinking Sociologically si legge che «oggi la sociologia è necessaria più di quanto lo sia mai stata in passato. Il compito in cui i sociologi sono esperti, quello di recuperare il legame perduto tra afflizione oggettiva ed esperienza soggettiva, è diventato più urgente $\mathrm{e}$ indispensabile che mai (1990a; trad. it. 2002: 251). Quasi tutti i suoi scritti sono dedicati alla fenomenologia delle forme di spaesamento, disgregazione, sfruttamento, emarginazione, sofferenza, ecc. e alla ricostruzione delle loro origini sociali. Sofia Capuano scrive che la sociologia di Bauman è critica anche perché continua «a domandarsi il motivo per cui il mondo è arrivato ad essere ciò che è» (2006: 402).

In questo interesse, di per sé, non vi è un orientamento pratico e morale. Le diagnosi possono essere e sono realizzate anche nel lavoro di consulenza, seppur con le cautele per ingerenze esterne, minori nella ricerca accademica. Bauman contesta la rappresentazione avalutativa della scienza ma rifiuta anche una sociologia che definisce idealmente ciò che è "normale" per poi constatarne fattualmente le difformità - il "modello durksoniano", di cui Habermas sarebbe un epigono. La critica si trova nel Terzo excursus di In Search of Politics (1999) nei termini di un rovesciamento prospettico. Non è il senso del normale che genera quello di crisi; è piuttosto l'idea di crisi che ci fa interrogare sulla norma: «Jürgen Habermas sosteneva che la percezione di un certo stato delle cose come "crisi" è una questione di teoria. Per parlare di crisi serve innanzitutto una teoria: l'immagine di uno stato delle cose normale, non problematico; la "crisi" insorge quando quello stato normale, usuale e familiare si sgretola [...] chiamiamo "crisi" una situazione in cui gli eventi sfidano qualunque cosa passi per normalità e le azioni routinarie non producono più i risultati cui eravamo abituati. Sul piano logico, il ragionamento di Habermas non fa una piega. Ma le vie intricate della nostra consapevolezza non seguono necessariamente indicazioni logiche. Per districarle sarebbe forse meglio invertire l'ordine del discorso e intonare la priorità concettuale dell'idea di "crisi" sulla percezione della "normalità". [...] Cerchiamo rifugio nella teoria quando cose che sono perfettamente alla nostra portata ci sfuggono di mano» (Ivi: 144).

Il giudizio si basa sulla sola consultazione di Legitimationsprobleme im Spätkapitalismus (1973), prescindendo da Theorie des kommunikativen Handelns, in cui figura la sistematizzazione delle patologie della riproduzione simbolica del mondo della vita: la perdita di senso, la carenza di legittimazione e la crisi di orientamento ed educazione, nella riproduzione culturale; l'insicurezza dell'identità collettiva, l'anomia e l'alienazione, nell'integrazione sociale; la rottura della tradizione, la carenza di motivazione e le psicopatologie, nella socializzazione (1981a; trad. it. 1986: 737). 
Bauman finisce per non cogliere appieno che, per Habermas, la "normalità" non è stabilita in astratto e arbitrariamente dal singolo studioso o da una parte o tutta la comunità scientifica e neppure viene desunta dalle rappresentazioni collettive. Quale sia lo stato normale si palesa nella ricostruzione razionale delle competenze cognitive, relazionali, motivazionali che consentono abitualmente la riproduzione del mondo della vita. Sono così ricostruiti differenti stati normali nello sviluppo ontogenetico delle persone e filogenetico della specie umana; senza prestabilire alcuna necessità perché le cose sarebbero potute svilupparsi in modo diverso e potrebbero mutare. Bauman non tiene sufficientemente in considerazione che la teoria habermasiana non presuppone assunti metafisici né filosofie della storia, bensì ricostruzioni razionali di competenze. A questa ricostruzione ex-post si accompagnano le ricerche empiriche delle scienze analitiche ed ermeneutiche sulle condizioni patologiche e gli effetti di colonizzazione del mondo della vita - la "burocratizzazione" della sfera privata e pubblica, ad opera del medium delle istituzioni pubbliche, e la "mercificazione" capitalistica di cose, eventi e persone tramite la forza gravitazionale del medium denaro. Per contro, nell'analisi habermasiana dei processi di riproduzione materiale dei sistemi sociali - economia e amministrazione - e delle loro crisi di crescita ed efficienza, la definizione dell'optimum e dei deficit di output sembra essere relativa alle aspettative degli attori, le quali differiscono in funzione di variabili storiche, ordinamenti socio-culturali e condizioni contestuali. L'opinione di Bauman che «l'idea di crisi è prima di tutto esperienziale, piuttosto che sistemica» (Beilharz 2000: 168) ci pare una conferma a favore di Habermas. Infine, lo studioso tedesco considera le crisi esogene e/o endogene dei principi di organizzazione delle differenti formazioni storico-sociali, che - in effetti - vengono definite "sistemiche" nel lessico funzionalista che Habermas adotta parzialmente nella teoria dell'evoluzione sociale. Così considerate le cose, l'ulteriore critica di Bauman, secondo cui la crisi è una "condizione normale", indicando essa uno stato di "transizione", ci sembra piuttosto congeniale a un discorso enfatico sull'ambivalenza.

Vi è, però, un secondo compito della sociologia, su cui i due studiosi sono concordi e che riguarda un altro significato del termine "chiarificazione", relativo alla qualità della riproduzione simbolica del mondo della vita, cioè ai processi di socializzazione degli individui, interazione sociale e trasmissione culturale. Habermas si riferisce all'impegno degli esperti e intellettuali nella sfera pubblica in discorsi teoretici, pragmatici, morali ed etici e critiche estetiche, in cui si tematizzano questioni di verità, efficienza, giustezza, preferibilità, gusto e autenticità. Adattati al linguaggio comune, i loro interventi contribuiscono a generare "prese di coscienza collettive", 
accrescere il capitale culturale e migliorare la qualità del sapere specialistico. Bauman comprese pienamente il trasferimento della "chiarificazione" sul piano pubblico, con cui lo studioso tedesco raccoglieva l'eredità francofortese - un tentativo parallelo al suo modo di recuperare il pensiero di Gramsci: «Quest'ultimo decisivo passo è stato compiuto da Jürgen Habermas - forse dal solo Habermas - nella sua recente reinterpretazione della concezione marxiana del rapporto tra conoscenza sociale e realtà sociale. Articolando la tradizione gramsciana del marxismo nel linguaggio della moderna scienza sociale, Habermas ha buone probabilità di far arrivare il messaggio a quel pubblico che ha considerato con equanimità offerte presentate in un linguaggio non familiare. Riallacciandosi direttamente alla sociologia moderna e ai suoi problemi più attuali, Habermas riafferma la tesi marxiana del processo di verità perché il corso della verifica della verità venga esteso al di là degli esperimenti di laboratorio gestiti da scienziati di professione, e si trasformi così nel processo di autenticazione» (1976b; trad. it. 1982: 187). In seguito, Habermas scriverà che il sapere specialistico deve favorire il "recupero critico-progettuale di un patrimonio condiviso" e non "cadere sulla testa" degli individui e dei gruppi (1988, trad. it. 1990: 37-38). La "perdita di senso", infatti, deriverebbe proprio da un impoverimento conseguente alla scissione tra le conoscenze coltivate dagli esperti e il sapere di senso comune che circola nel mondo quotidiano (1981a; trad. it. 1986: 990). Per altro verso, quanto più il sapere specialistico si isola e rimane élitario, tanto maggiore è il rischio di una "musealizzazione" di conoscenze e pratiche "sterilizzate", prive di vitalità e rigidamente parcellizzate negli ambiti del lavoro accademico e intellettuale (1989, trad. it. 1990: 94). Coloro che detengono istituzionalmente l'autorità scientifica e intellettuale sul sapere ricoprono certo un ruolo attivo primario e, sovente, riconosciuto ma, nel dialogo tra gli esperti e i pubblici, i primi non dispongono dell'ultima parola perché le loro conoscenze e opinioni devono passare il "test di accettazione" dei secondi. Bauman concorda, affermando che essi «non controllano il processo in modo monopolistico. Né il loro verdetto, sostenuto unicamente in termini di discorso propriamente detto, si può considerare finale e definitivo, a meno che non sia "autenticato"» (1976b; trad. it. 1982: 190). Aver differenziato le "giustificazioni ragionate", cui mira il discorso, e le autenticazioni della critica (dialogo), «mette Habermas in una posizione a parte rispetto a tutti i sociologi precedentemente presi in esame» (Ibidem): Ernst Bloch, Edgar Morin, Henry Kariel e Manfred Stanley.

Habermas e Bauman erano, altresì, accomunatati, almeno negli anni Settanta, dal recupero della critica marxiana dell'ideologia, di «interessi, che piegano la coscienza sotto il dominio delle cose e delle connessioni reifica- 
te $[\ldots]$ interessi materiali, ancorati a configurazioni storicamente specifiche del lavoro alienato, delle soddisfazioni negate, e della libertà soppressa» (1963; trad. it. 1973: 396-397; cfr. Bauman, 1976b; trad. it. 1982: 166-167). Habermas definì l'ideologia un'“autoillusione divenuta oggettiva" (1971a; trad. it. 1973: 45) e, più compiutamente, in scritti successivi, una "parvenza oggettiva" (1981a, trad. it. 1986: 988). La formula contiene due aspetti: l'antitesi tra il "sapere fondato" e le "false rappresentazioni" e la "non casualità" delle distorsioni, a cui sarebbero soggetti - "inconsapevolmente" tutti coloro che interpretano il mondo alla luce di "categorie ideologiche" che assicurano la riproduzione non problematica di sistemi sociali, in cui sono istituzionalizzati la partecipazione differenziata al potere politico, la redistribuzione diseguale della ricchezza economica, il riconoscimento selettivo del prestigio sociale e della dignità culturale di specifici stili di vita. Rispetto alle costrizioni esterne manifeste - intimidazioni, incentivi e altre forme di violenza - e alle manipolazioni volontarie ma latenti, compiute per celare interessi strategici, la cui conoscenza pubblica indurrebbe le controparti a rigettare il consenso, la critica dell'ideologia fa riferimento alle "costrizioni interne": situazioni di autoinganno dei soggetti che rimandano alle condizioni strutturali della "comunicazione sistematicamente distorta".

Qui risiede l'originalità della proposta di Habermas, che Bauman segue, con cui egli ha inteso superare i "dilemmi" della Scuola di Francoforte. La questione riguarda il fondamento normativo di una sociologia critica, dal punto di vista della "prassi", l'altra principale direttrice dell'intera opera e principale motivo di rottura, assieme alla critica del "concetto di verità" e del "rapporto con le scienze" e alla loro sottovalutazione delle tradizioni democratiche dello stato di diritto (1981b; trad. it. 1983: 223-232). Habermas ritiene che la linea di pensiero che accomuna Marx, Weber, Lukács, Adorno e Horkheimer, e che egli intende "portare a compimento" (1999c, trad. it. 2001: 317), non era stata in grado di risolvere il "corto circuito" tra ragion pratica e prassi di vita, ricadendo nel "trilemma" che impedisce di affidarsi, per determinare i contenuti normativi, alla "filosofia della storia", alla "costituzione antropologica dell'uomo" e al «fortunato repertorio ereditato dalle tradizioni culturali occidentali» (1992, trad. it. 1996: 10). Lo scacco impone il ripensamento della critica dell'ideologia e lo condurrà a trovare una via d'uscita dall'impasse" con l'applicazione delle scienze ricostruttive ai processi ontogenetici e filogenetici (1976, trad. it. 1979: 197).

Sul finire degli anni Sessanta, Habermas poneva ancora il problema ricorrendo al nesso tra autoriflessione e interesse all'emancipazione, e a tale progetto si riferisce Bauman in Towards a Critical Sociology. Il sociologo polacco seguiva passo dopo passo la replica con cui Habermas aveva rispo- 
sto alle obiezioni «contro la tesi, secondo la quale, nella comprensione che si produce attraverso l'autoriflessione, la conoscenza e l'interesse conoscitivo all'emancipazione facciano tutt'uno» (1971a; trad. it. 1973: 45). Si trattava di spiegare come fosse possibile che - e se necessariamente - la chiarificazione operi nel senso di realizzare dei fini emancipativi «che esigono una presa di posizione rischiosa e proprio per questo l'abbandono del ruolo neutrale di colui che prende parte al discorso» (Ivi: 46).

Egli accettava, in primo luogo, la diversificazione dei due interessi conoscitivi "inferiori" - tecnico e pratico - e la descrizione dell'interesse conoscitivo emancipativo, a partire dall'intrinseco collegamento all'azione: «il dialogo che deriva dall'interesse emancipatorio non può in nessuna fase essere staccato dal suo impegno pratico nella comunicazione, nel processo della vita. Esso non si limita all'obiettivo della giustificazione ragionata; vuole anche mettere alla prova se stesso nell'effettiva accettazione della sua soluzione ipotetica nella prassi dei partner» (1976b; trad. it. 1982: 189).

Nel passaggio ulteriore viene affermato che l'interesse emancipativo non è una "caratteristica extra-temporale" propria della condizione umana. Ricorrendo alla figura di pensiero dell'antitesi, Habermas sostiene che esso si forma e definisce solo in presenza dell'esperienza concreta e opposta di "dominio", che investe le credenze, le condotte e i vissuti di soggetti individuali e collettivi e «porta alla coscienza quelle determinanti di un processo di formazione che determinano ideologicamente una prassi attuale dell'azione e della concezione del mondo» (1971a; trad. it. 1973: 55). Questa condizione di dominazione può essere sia materiale che simbolica ed è a quest'ultima forma che lo studioso tedesco - e di conseguenza Bauman rivolgono unicamente la loro attenzione, identificandola con una sistematica distorsione della situazione comunicativa ideale. L'interesse all'emancipazione si formerebbe «solo nella misura in cui un potere repressivo viene posto durevolmente sotto forme di esercizio normativo della forza nelle strutture di una comunicazione distorta, viene cioè istituzionalizzato come dominio» (Ivi: 54; Bauman, 1976b; trad. it. 1982: 188). Egli riduce la distorsione comunicativa a un'asimmetria tra le posizioni di potere dei partecipanti la cui durata struttura in modo sistematico istituzioni fondate e generatrici di dominio (Ivi, 188-189; sul concetto cfr. Corchia, 2010: 87-93).

Poiché la comunicazione non distorta implica il disvelamento delle espressioni con cui i soggetti illudono gli altri e se stessi, Habermas aveva proposto, in terzo luogo, il parallelismo tra critica e colloquio psicoanalitico. Non c'è modo qui di ricostruire tutti gli aspetti di una riflessione su cui egli fornirà precisazioni e ripensamenti (cfr. Corchia, 2007b: 383-384). Rileggendo l'Introduzione alla seconda edizione di Theorie und Praxis, Bau- 
man mostra attenzione verso l'analogia psicoanalisi e teoria critica, a cui la prima fornisce un modello per il dialogo attivato dall'interesse emancipatorio. Ciò che lo convince meno non è tanto il parallelo ma che Habermas vi colleghi la questione dell' "organizzazione" del processo di chiarificazione.

Come è evidente, indicare le condizioni di esistenza della critica non significa chiarire - questa era la questione - come sia possibile che l'autoriflessione operi nel senso di realizzare dei fini emancipativi. Perché la coscienza del dominio subito non potrebbe generare accettazione? La risposta di Habermas è stata descritta da Lucio Cortella: l'interesse emancipativo che «si prolunga nella sfera teorica, sicché solo grazie a esso diventa possibile quel processo riflessivo di distanziamento dal dominio e dall'ideologia che consiste nella critica di ogni costrizione sociale, normativa e culturale» sarebbe «già attivo nella prassi dei soggetti storico-sociali» (2015: 341). Più precisamente, allora, Habermas riteneva che l'attivazione dell'interesse all'emancipazione, nei processi individuali e collettivi di autoriflessione, sia fondata e ancorata in una «motivazione che è altrettanto generalizzata quanto gli altri due interessi conoscitivi, che pure dal punto di vista antropologico sono radicati più profondamente» (1971a; trad. it. 1973: 58n).

Veniva, da ultimo, introdotta la distinzione tra "autoriflessione" e "ricostruzione" dei sistemi di regole e competenze che dobbiamo presupporre nelle nostre esperienze del mondo naturale, sociale e interiore (Ivi: 55). Per la prima volta in forma compiuta - fatti salvi alcuni spunti presenti in Der Universalitätsanspruch der Hermeneutik (1970) - Habermas presentava l'idea di una scienza ricostruttiva che definirà il suo programma di ricerca. Egli scriveva che l'interesse emancipativo entra "immediatamente" nell'autoriflessione ma quest'ultima non fornisce alcuna fondazione della critica. Solo basandosi sulle "ricostruzioni" è possibile una elaborazione teorica dell'origine di tale interesse e, viceversa, solo attraverso il "rapporto indiretto" con l'autoriflessione le ricostruzioni entrano in contatto con l'interesse conoscitivo all'emancipazione (1971a; trad. it. 1973: 57). Inoltre, anticipando un'interpretazione sviluppata alcuni anni dopo, si legge che la ricostruzione delle condizioni di genesi dell'interesse emancipativo costituisce il nucleo del materialismo storico di Marx, se inteso come teoria dell'evoluzione sociale (Ivi: 29). Questa proposta non è stata raccolta da Bauman, più interessato alle brevi digressioni di Habermas sull'organizzazione del rapporto tra teoria scientifica del capitalismo e presa di coscienza della classe operaia (Ivi: 64-65; Bauman, 1976b; trad. it. 1982: 192). In Teoria critica, redatto nei primi anni Ottanta, Bauman continuava ad attribuire ad Habermas, rispetto alla "scienza ordinaria", solo il merito di aver costruito un secondo "ponte" che unisce la teoria e la pratica critica, con un 
concetto di interesse all'emancipazione ancorato nella storia. Esso è il prodotto di condizioni particolari, non universali per la specie, che richiedono l'esercizio di riflessività e argomentazione (1991b; tr. it. 2005: 183-185).

L'apprezzamento nei confronti di Habermas, in quegli anni, era indubbio. Per tale ragione, Dennis Smith riteneva "non irragionevole" supporre che Bauman avesse maggiore affinità con l'epigono della "seconda generazione" della Scuola di Francoforte piuttosto che con i fondatori. L'interesse però svanì completamente: «Per diversi anni Bauman ha trovato nel modello di Habermas del "discorso ideale" e della "comunicazione senza distorsioni" un prezioso supporto intellettuale per le proprie aspirazioni utopiche. Tuttavia, ad un certo punto, negli anni ' 80 , Bauman ha compiuto un cambio di lealtà [switch of loyalty] da Habermas verso Adorno» (1999: 175).

Una delle principali ragioni del distacco viene rintracciata nella sfiducia verso la possibilità di realizzare le condizioni del dialogo nella sfera pubblica. Legislators and Interpreters - che riscosse grande eco nella comunità internazionale - è il luogo in cui si consuma in pieno il ripensamento. Habermas finisce nella tenaglia del dualismo modernità-postmodernità con cui Bauman reinterpreta la storia, i vecchi e i nuovi compiti degli intellettuali. Essi sono considerati gli "architetti" del senso moderno di cittadinanza, hanno contribuito a legittimare gli sforzi dello stato moderno per controllare e civilizzare la popolazione su cui esercitava sovranità e, in tal senso, Bauman attribuiva a quel tipo di intellettuale il ruolo di "legislatore". Nella ricostruzione storica della loro affermazione, a partire dall'"epoca dei lumi”, qui, egli non si avvale di Strukturwandel der Öffentlichkeit (1962) che sarà tradotto in inglese tardivamente, nel 1989. Il testo gli era comunque noto, come risulta dal saggio Ideology and the Weltanschauung of the Intellectuals, che anticipa brani ripresentati nel volume: «Questo fenomeno ha ricevuto la più completa analisi, sino ad oggi, nell'impressionante studio di Jürgen Habermas sulla struttura della sfera pubblica» (1983b: 114). Lo studioso tedesco viene però considerato un esempio inattuale del modello illuminista di un intellettuale che esercita la propria vocazione in «un ambito separato dal resto della popolazione», in ideali "sociétés de pensée", tra i pari, a cui è riconosciuto «il diritto (e il dovere) di rivolgersi alla nazione in nome della Ragione, ponendosi al di sopra delle divisioni e degli interessi materiali di parte» e le cui affermazioni possiedono «la veridicità esclusiva e l'autorità morale che solo un tale ruolo di portavoce può dare» (1987; trad. it. 1992: 32). Questa comunità di philosophes fu generata e si riproduce nella discussione ed esclude ogni ingerenza esterna nella determinazione del consenso, secondo il principio che «nessun potere, divino o terreno, nessuna differenza di status sociale tra i partecipanti, nessuna risorsa eco- 
nomica o politica possa influire sul risultato della discussione; l'unico potere al quale si può far appello, e su cui ci si può fondare per raggiungere un consenso valido, deve essere quello dell'argomentazione» (Ivi: 114). Le "norme controfattuali" presupposte implicitamente nell'agire comunicativo e formalizzate nel discorso sono etichettate come «la famosa utopia della "comunicazione non distorta" di Jürgen Habermas» (Ivi: 48). Bauman precisava che la "comunicazione non distorta" va considerata come un modello euristico con cui criticare e dimostrare o meno valido il consenso fattualmente raggiunto. Non si tratta di «una proposta pratica per raggiungere un consenso nel nostro proprio mondo» - come frainteso da molti critici ai quali «sembrava una idea nebulosa, talmente in contrasto con l'esperienza del dibattito pubblico nel secolo ventesimo da porre l'immagine della comunicazione non distorta sullo scaffale su cui raccolgono polvere altri bei sogni di saggi pieni di buona volontà» (Ivi: 114-115). Tuttavia, quella pretesa di ricostruire nella comunità scientifica le condizioni astratte e universali per "la ricerca laica della verità" colloca Habermas tra i legislatori. Ciononostante, anch'egli aveva maturato - così valutava Bauman - la «consapevolezza profonda che le ambizioni che fondavano la validità del modo di vita intellettuale sono fallite» (Ivi: 170). L'anno seguente, nel saggio Is There A Postmodern Sociology?, Bauman ribadirà che le tesi di Legitimationsprobleme im Spätkapitalismus avevano avuto il senso della percezione intellettuale di una crisi causata dall'evidente minor rilievo del discorso sulla legittimazione per le strutture di dominio: «La crescente irrilevanza della legittimazione ha coinciso con la crescente libertà di dibattito intellettuale. Si sospetta più di una coincidenza» (1988a: 98).

La decisione di tenere in vita un progetto illuministico finirebbe per tradursi in una "resa finale" e sarebbe incompatibile con il riconoscimento della «pluralizzazione di discorsi contestualizzati comunitariamente e tradizionalmente, che reclama quelle localizzazioni di verità, giudizio e gusto che la modernità negava e si sforzò di superare» (1987; trad. it. 1992: 148). Nella postmodernità sarebbe rimasta solo l'opzione di una strategia che abbandona le ambizioni legislative, l'attaccamento ai discorsi di legittimazione e alla ricerca dei fondamenti, e accetta la diversità di codici culturali. Conseguentemente, anche il ruolo dell'intellettuale sarebbe mutato da "legislatore" a "interprete" - un mediatore tra differenti culture: «la visione del mondo tipicamente postmoderna è, in linea di principio, quella di un numero illimitato di modelli di ordine, ciascuno generato da una serie di pratiche relativamente autonome [...] Ciascuno dei tanti modelli d'ordine ha un senso soltanto nei termini delle pratiche che lo convalidano [...] La strategia tipicamente postmoderna del lavoro intellettuale è quella caratte- 
rizzata nel modo migliore dalla metafora del ruolo di "interprete". Esso consiste nel tradurre affermazioni, fatte all'interno di una tradizione fondata sulla comunità, in modo tale che possano essere capite all'interno del sistema di conoscenza basato su di un'altra tradizione» (Ivi: 14-15).

Ci limitiamo qui soltanto a esprimere il dissenso nei confronti di Bauman, la cui interpretazione, anche su tale aspetto, deriva da fraintendimenti. Ciò che viene eluso è la distinzione che Habermas compie tra gli "apriori semantici" e gli "apriori pragmatici" (1981a; trad. it. 1986: 455), con la composizione, nel lessico di Bauman, dell'antinomia tra l'approccio ermeneutico dello "storicismo" e quello ricostruttivo del "trascendentalismo". La distinzione fra la semantica e la pragmatica mette in risalto la differenza tra gli aspetti "particolari" e gli aspetti "universali" del processo di intesa. Più precisamente, gli apriori semantici si incarnano nel "sapere linguistico" e nel "senso comune" - oggetto delle scienze comprendenti - e si diversificano nelle molteplici culture, mentre gli apriori pragmatici - oggetto delle scienze ricostruttive - sono relativi alle condizioni formali necessarie all'esperienza del mondo ("condizioni pragmatiche dell'oggettività") e alle condizioni formali necessarie all'accettabilità delle argomentazioni su qualcosa nel mondo ("condizioni comunicative della razionalità delle pretese di validità”) (Habermas, 1999a; trad. it. 2001: 87-88; Corchia, 2010: 13-110). Da questo punto di vista, le posizioni di Habermas sono meno incompatibili con l'ermeneutica di quanto Bauman e suoi interpreti siano disposti ad ammettere. Del resto, l'analogia con il lavoro di traduzione è ampiamente utilizzata dall'allievo e collega di Hans-Georg Gadamer e, non dissimilmente dal sociologo della liquidità, negli scritti troviamo l'idea della "solidarietà tra estranei" che accompagna e orienta la "fusione di orizzonti".

Un'ulteriore convergenza sui compiti della teoria critica, infine, mi è stata suggerita dall'ascolto della lezione sulla "sociologia del possibile" di Paolo Jedlowski. Riguarda l'apertura delle prospettive sul mondo, ossia quel "progettare alternative all'esistente" - scrive Habermas - che Bauman definisce parte del "pensare sociologicamente": «Si potrebbe dire che il servizio più importante che l'arte di pensare sociologicamente può rendere a ciascuno di noi, consista nel renderci più sensibili; può acuire i nostre sensi, spalancare i nostri occhi in modo che noi possiamo esplorare le condizioni dell'uomo per troppo tempo rimaste poco visibili» (1990a; trad. it. 2000: 15). Habermas ritiene che, in tempi, come in nostri, in cui vi è un "ingorgo di futuro", la critica debba stimolare la ricerca di senso per "ciò che manca" e per "ciò che potrebbe essere altrimenti" (2006, trad. it. 2011: 11). Sottolineiamo la concordanza con il pensiero di Bauman: «La sociologia offre un commento informato, saggio, illuminato sull'esperienza attua- 
le, un commento che interagisce con questa esperienza e la rende quindi più ricca, ampliandone gli orizzonti. Il compito della sociologia oggi è quello di scalzare le certezze, perché ogni certezza infiacchisce le scelte. Sono d'accordo con Walter Benjamin che la storia è la tomba delle possibilità. Ogni sviluppo storico significa che diverse possibilità sono state uccise, e la sociologia si preoccupa di tenere le possibilità vive» (1992b; trad. it. 2005: 45). Lasciamo cadere, qui, il riferimento implicito alle tesi sulla filosofia della storia di Benjamin a cui Habermas ha dedicato passaggi molto suggestivi sulla "rammemorazione" come "salvamento di momenti messianici".

$\mathrm{Ci}$ preme piuttosto sottolineare come i due studiosi mantengano vivo persino un concetto di "utopia" che non concerne tanto la speranza o il pessimismo, bensì la coscienza del tempo. Nel libro-intervista con Keith Tester, riprendendo riflessioni già espresse negli scritti del primo periodo inglese, in particolare in Socialism: The Active Utopia (1976a), Bauman faceva ancora coincidere i compiti della sociologia critica con un socialismo che rimane fecondo solo finché si mantiene nel "regno del possibile" (2001; trad. it. 2002: 154). Nel discorso tenuto da Habermas all'assemblea del parlamento spagnolo, il 26 novembre 1984, ritroviamo l'impellenza del pericolo di un "esaurimento delle energie utopiche", annunciato dalle inquietudini della coscienza storica di fronte alla "contrazione dell'orizzonte futuro" (1985, trad. it. 1998: 14). È degno di nota che proprio interrogandosi sul tramonto dell'utopia socialista di una società più umana, egualitaria e liberata dal bisogno, Habermas indicava come ultima chance l'utopia di una "prassi comunicativa quotidiana non reificata", che presuppone le condizioni formali e materiali della "situazione linguistica ideale" (Ivi: 52).

Concludiamo questo iniziale tentativo di ricostruzione filologica e tematica del confronto tra i due studiosi con una suggestione scaturita dalla lettura del capitolo Thinking in Dark Times di Liquid Life (2005), una versione modificata del saggio di due anni prima, tradotto anche in italiano, da Micromega, con il titolo Adorno e la globalizzazione (2003). Bauman conclude la "rivisitazione" di Adorno e Hannah Arendt, interrogandosi sulla crisi attuale e sul futuro degli intellettuali, un tempo «custodi delle speranze e promesse disattese del passato e critici di un presente colpevole di aver dimenticato e soppresso tali aspirazioni» (2005; trad. it. 2006: 166). Il testo approfondisce le riflessioni che Bauman svolse nella lettura, presso la Paulskirche di Francoforte a.M. per il conferimento del Theodor W. AdornoPreis - sulla cui assegnazione Habermas, all'epoca, aveva molta influenza. In quell'occasione, Bauman si definì "discepolo" di Adorno, così motivando le ragioni gnoseologiche dei sentimenti di affinità spirituale e parentela temperamentale: «Adorno sapeva - a differenza di tanti altri che, per amore 
della coscienza pulita e dell'equilibrio mentale, non sanno o non ammettono di sapere o non vorrebbero sapere - che non ci sono, né ci dovrebbero essere, risposte dirette a domande contorte, né ci sono soluzioni chiare a situazioni ambivalenti. Sapeva che chi pensa e si preoccupa è destinato a navigare tra la Scilla del pensiero puro ma impotente e le Cariddi di un'efficace ma inquinata offerta di dominio. Terzium non datur» (1998b).

Tornando alla questione degli intellettuali, Bauman ricorda che è opinione comune - formulata probabilmente da Habermas per la prima volta che la risposta di Adorno a domande del genere trovi la "migliore espressione" nell'immagine del "messaggio nella bottiglia" (2003; trad. it. 2006: 167). L'allegoria della Flaschenpost fu formulata, in effetti, da Adorno nella Philosophie der neuen Musik, a proposito della condizione avanguardistica ed era frequente nelle conversazioni con Horkheimer, nell'esilio di Santa Monica, durante la stesura dei Philosophical Fragments, dal 1942 al 1944, pubblicati ad Amsterdam dalla Querido, con il titolo Dialektik der Aufklärung (1947). Le scarse attese sulla risonanza di quel messaggio nella cultura di massa si ritrovano nel quasi excipit del paragrafo Propaganda: «Se il discorso, oggi, deve rivolgersi a qualcuno, non è già alle cosiddette masse, né al singolo, che è impotente, ma piuttosto a un testimone immaginario, a cui lo lasciamo in eredità perché non scompaia interamente con noi» (1947; trad. it. 1966: 272; cfr. Müller-Doohm, 2003; trad. it. 2003: 373-386). Con tale pensiero anticipatamente rammemorante e portato alla forma ineguagliata nelle Meditazioni della vita offesa (1951; trad. it. 1954), Bauman identifica la sua "equazione" composta quasi esclusivamente da incognite, di cui può dire, con Karl Marx: Dixi et salvavi animam meam.

L'impresa testimonia che la frustrazione è transitoria e la speranza duratura, ma parlando dei contenuti del messaggio nella bottiglia, laconicamente, egli si interroga sulle raccomandazioni che gli intellettuali della sua generazione - contigua a quella di Adorno - possono dispensare rispetto alle sfide e ai compiti che una nuova generazione e i suoi intellettuali dovranno affrontare: «Noi avvertiamo, intuiamo, sospettiamo ciò che occorre fare. Ma non possiamo conoscere i contorni e la forma che assumerà. E, tuttavia, possiamo esser certi che la forma non ci apparirà familiare. Essa sarà diversa da tutto ciò che è per noi consueto» (2003; trad. it. 2006: 179-180). Con meno enfasi, è pensiero non estraneo ad Habermas, addestrato nella palestra della critica della filosofia della storia, secondo il quale nei fatti umani nulla è predefinito e tutto potrebbe essere diverso (Corchia, 2007: 60-81). Ciò che distanzia la prospettiva di Habermas da quella di Bauman, in definitiva, è stato provocatoriamente espresso da Peter Beilharz nell'introduzione a The Bauman Reader, scrivendo che «La modernità - almeno su 
questo punto Bauman mostra affinità con Habermas - è tuttora un progetto, non realizzato, ma ancora tale, perché irrealizzabile (Beilharz, 1994, 1997).

\section{Riferimenti bibliografici}

Adorno Th.W. (1951). Trad. it. Minima moralia. Meditazioni della vita offesa. Torino: Einaudi, 1954.

Adorno Th.W., Horkheimer M. (1947). Trad. it. Dialettica dell'illuminismo. Torino: Einaudi, 1966.

Almeida F.Q. (2015). Bauman entre Habermas e Richard Rorty: interpretações... Impulso, 25, 64: 53-65.

Bauman Z. (1973). Trad. it.: Cultura come prassi. Bologna: il Mulino, 1976.

Bauman Z. (1976a). Socialism. The Active Utopia. New York: Holmes and Meier Publishers.

Bauman Z. (1976b). Trad. it. Critica del senso comune. Verso una nuova sociologia. Roma: Editori Riuniti, 1982.

Bauman Z. (1976c). J. Habermas, Legitimation Crisis. New Society, 38, 733: 147-148.

Bauman Z. (1978). Hermeneutics and Social Science: Approaches to Understanding. London: Hutchinson.

Bauman Z. (1982). Trad. it.: Classi: prima e dopo. In Bauman Z., Globalizzazione e glocalizzazione (pp. 88-126). Roma: Armando, 2005.

Bauman Z. (1983a). The Politics of Social Theory: Habermas, Freud and the Critique of Positivism by Russell Keat. The British Journal of Sociology, 34, 1: 154-156.

Bauman Z. (1983b). Ideology and the Weltanschauung of the Intellectuals. Canadian Journal of Political and Social Theory, 7, 1-2: 104-117; repr. 15, 1-3, 1991: 107120.

Bauman Z. (1987). Trad. it.: La decadenza degli intellettuali. Da legislatori a interpreti. Torino: Bollati Boringhieri, 1992.

Bauman Z. (1988a). Is There A Postmodern Sociology?. Theory, Culture, and Society, 5, 2: 217-237; repr. In Z. Bauman, Intimations of Postmodernity (pp. 93-113). London-New York: Routledge, 1992.

Bauman Z. (1988b). The Philosophical Discourse of Modernity by Jürgen Habermas. Sociology, 22, 3: 473-475.

Bauman Z. (1989a). Trad. it.: Modernità e Olocausto. Bologna: il Mulino, 1992.

Bauman Z. (1989b). Hermeneutics and Modern Social Theory. In D. Held, J.B. Thompson (eds.), Social Theory of Modern Societies: Anthony Giddens and His Critics (pp. 34-55). Cambridge: Cambridge University Press.

Bauman Z. (1989c). Sociological Responses to Postmodernity. Thesis Eleven, 23, 3563; repr. in Z. Bauman, Intimations of Postmodernity (pp. 26-67). London-New York: Routledge, 1992.

Bauman Z. (1990a). Trad. it.: Pensare sociologicamente. Napoli: Ipoermedium, 2000. 


\section{Corchia}

Bauman Z. (1990b). Philosophical Affinities of Postmodern Sociology. The Sociological Review, 38, 3: 411-444; repr. in Z. Bauman, Intimations of Postmodernity (pp. 114-148). London-New York: Routledge, 1992.

Bauman Z. (1991a). Trad. it.: Modernità e ambivalenza. Torino: Bollati Boringhieri, 2010.

Bauman Z. (1991b). Trad. it.: Teoria critica. In Bauman Z., Globalizzazione e glocalizzazione (pp. 164-188). Roma: Armando, 2005.

Bauman Z. (1992a). Intimations of Postmodernity. London-New York: Routledge.

Bauman Z. (1992b). Trad. it.: L'intervista di Telos. In Bauman Z., Globalizzazione e glocalizzazione (pp. 33-46). Roma: Armando, 2005.

Bauman Z. (1992c). Appendix: Sociology, postmodernity and exile: an interview with Zygmunt Bauman. An interview by Richard Kilminster and Ian Varcoe. In Id., Intimations of Postmodernity (pp. 205-228). London-New York: Routledge.

Bauman Z. (1993). Trad. it.: Modernità. In Bauman Z., Globalizzazione e glocalizzazione (pp. 190-198). Roma: Armando, 2005.

Bauman Z. (1995). Trad. it. parz. La società dell'incertezza. Bologna: il Mulino, 1999.

Bauman Z. (1998a). Trad. it.: Dentro la globalizzazione. Le conseguenze sulle persone. Roma-Bari: Laterza, 1999.

Bauman Z. (1998b). Ein Mensch, der denkt, zwischen Scylla und Charybdis: die Rede von Zygmunt Bauman, der [am 13. Sept. 1998] in der [Frankfurter] Paulskirche den Theodor W. Adorno-Preis erhielt. Frankfurter Rundschau, 54, 213: 12.

Bauman Z. (1999). Trad. it. La solitudine del cittadino globale. Milano: Feltrinelli, 2000.

Bauman Z. (2003). Trad. it. Pensare in tempi oscuri (rileggendo Arendt e Adorno). In Id., Vita liquida (pp. 153-182). Roma-Bari: Laterza, 2006.

Bauman Z. (2005). Trad. it. Vita liquida. Roma-Bari: Laterza, 2006.

Bauman Z. (2008). Trad. it. La paura e la responsabilità. Incontro con Zygmunt Bauman di Emanuele Caroppo e Patrizia Brogna. AREL la rivista, 2: 161-165.

Bauman Z., Tester K. (2001). Trad. it. Società, etica, politica. Conversazioni con Zygmunt Bauman. Milano: Cortina, 2002.

Beilharz P. (2000). Zygmunt Bauman: Dialectic of Modernity. London: Sage.

Beilharz P. (2001). Trad. it.: Introduzione di Peter Beilharz: approccio a Zygmunt Bauman. In Bauman Z., Globalizzazione e glocalizzazione (pp. 13-32). Roma: Armando, 2005.

Beilharz P. (2011). Zygmunt Bauman. In G. Ritzerm J. Stepnisky (eds.), The WileyBlackwell Companion to Major Social Theorists (pp. 155-174). Malden (MA): John Wiley \& Sons.

Bernstein R.J. (ed.) (1985). Habermas and Modernity. Cambridge (Ma): MIT Press.

Best S. (2013). Zygmunt Bauman: Why Good People Do Bad Things. Farnham: Ashgate.

Blackshaw T. (2005). Zygmunt Bauman (Key Sociologists). London/New York: Routledge.

Blackshaw T. (ed.) (2016). The New Bauman Reader: Thinking Sociologically in Liquid Modern Times. Manchester: Manchester University Press. 


\section{Corchia}

Bordoni C. (ed.) (2016). Zygmunt Bauman. With an original contribution. Bruxelles: Revue Internationale de Philosophie, 70, 3.

Bovone L. (2002). Dalla morale alla comunicazione?: la sociologia come racconto di racconti. Studi di Sociologia. 40, 4: 383-411; repr. in Bovone L., Tra riflessività e ascolto: l'attualità della sociologia (pp. 69-104). Roma: Armando, 2010.

Caccamo De Luca R. (1982). Introduzione. L'itineriario sociologico di Zygmunt Bauman. In Z. Bauman, Critica del senso comune. Verso una nuova sociologia (pp. 727). Roma: Editori Riuniti.

Cannon B. (2014). Towards a Theory of Counter-Modernity: Rethinking Zygmunt Bauman's Holocaust Writings. Critical Sociology, 42, 1: 49-69.

Capuano S. (2006). Bauman e il dibattito moderno. In M.A. Toscano (a cura di), Introduzione alla sociologia (pp. 394-409). Milano: FrancoAngeli.

Chardel P.-A. (2013). Zygmunt Bauman. Les illusions perdues de la modernité. Paris: CNRS Editions.

Cipriani G. (2013). Il rischio nella società sociologica contemporanea: Zygmunt Bauman, Niklas Luman e Ulrich Beck. Macerata: Edizioni simple.

Conte G. (2011). Per una ricostruzione filologica e filosofica di Bauman: Dal marxismo degli esordi alla teorizzazione della società liquida. i-lex, 13-14, 121-140.

Corchia L. (2007a). Il programma di ricerca e la sua recezione critica. In M. Ampola, L. Corchia, Dialogo su Jürgen Habermas. Le trasformazioni della modernità. (pp. 786). Pisa: ETS, $2010^{2}$.

Corchia L. (2007b). Teoria sociale e prassi di vita. In M. Ampola, L. Corchia, Dialogo su Jürgen Habermas. Le trasformazioni della modernità (pp. 377-392). Pisa: ETS, $2010^{2}$.

Corchia L. (2009). La teoria della socializzazione di Jürgen Habermas. Un'applicazione ontogenetica delle scienze ricostruttive. Pisa: ETS, $2012^{2}$.

Corchia L. (2010). La logica dei processi culturali. Jürgen Habermas tra filosofia e sociologia. Genova: ECIG.

Corchia L. (2017). I compiti di una teoria critica della società. Il percorso intellettuale di Jürgen Habermas. Rassegna Italiana di Sociologia, 58, 1: 5-30.

Cortella L. (2015). Paradigmi di teoria critica. Politica \& Società, 4, 3: 333-354.

Coser L.A. (1989). Legislators and Interpreters: On Modernity, Post-Modernity and Intellectuals by Zygmunt Bauman. The Canadian Journal of Sociology, 14, 2: 263 264.

D'Andrea F. (2006). Conoscenza antropologica, figure dell'individualismo e autoriflessione dell'occidente. In A. De Simone, F. Di Clemente, F. D'andrea, F. Fornari, Tra Dilthey e Habermas. Esercizi di pensiero su filosofia e scienze umane (pp. 525604). Perugia: Morlacchi.

Davis M. (2008). Freedom and Consumerism: A Critique of Zygmunt Bauman's Sociology. Aldershot: Ashgate.

Davis M. (ed.) (2013). Liquid Sociology: Metaphor in Zygmunt Bauman's Analysis of Modernity. Farnham: Ashgate.

Davis M., Tester K. (eds.) (2010). Bauman's Challenge: Sociological Issues for the 21st Century. Basingstoke: Palgrave Macmillan. 


\section{Corchia}

Dawson M. (2015). Sociology as Conversation: Zygmunt Bauman's Applied Sociological Hermeneutics. Sociology, 49, 3: 582-587.

Docherty Th. (1994). Postmodernist theory: Lyotard, Baudrillard and others. In R. Kearney (ed.), Routledge History of Philosophy, Volume VIII. Twentieth-Century Continental Philosophy (pp. 392-416). London-New York: Routledge.

Elliott A. (ed.) (2007). The Contemporary Bauman. London: Routledge.

Giaccardi C., Magatti M. (2001), La globalizzazione non è un destino. Mutamenti strutturali ed esperienze soggettive nell'età contemporanea. Roma-Bari: Laterza.

Giacomantonio F. (2014). Sociologia dell'agire politico. Bauman, Habermas, Žižek. Roma: Edizioni Studium.

Habermas J. (1962). Trad. it. Storia e critica dell'opinione pubblica. Bari: Laterza, 1971.

Habermas J. (1963). Trad. it. Prassi politica e teoria critica della società. Bologna: il Mulino, 1973.

Habermas J. (1968). Trad. it.. Conoscenza e interesse. Bari: Laterza, 1970.

Habermas J. (1970). Trad. it. La pretesa di universalità dell'ermeneutica. In AA.VV., Ermeneutica e critica dell'ideologia (pp. 131-167). Brescia: Queriniana, 1979.

Habermas J. (1971a). Trad. it. Introduzione: la difficile mediazione tra teoria e prassi. In Id., Prassi politica e teoria critica della società (pp. 29-75). Bologna: il Mulino, 1973.

Habermas J. (1971b). Trad. it. Osservazioni propedeutiche per una teoria della competenza comunicativa (elaborate per la discussione di un seminario). In Id., N. Luhmann, Teoria della società o tecnologia sociale (pp. 67-94). Milano: Etas Kompass, 1973.

Habermas J. (1973). Trad. it. La crisi della razionalità nel capitalismo maturo. Bari: Laterza, 1975.

Habermas J. (1976). Trad. it.. Per la ricostruzione del materialismo storico. Milano: Etas libri, 1979.

Habermas J. (1981a). Trad. it. Teoria dell'agire comunicativo. Bologna: il Mulino, 1986.

Habermas J. (1981b). Trad. it. Dialettica della razionalizzazione: J. Habermas a colloquio con A. Honneth, E. Knödler-Bunte e A. Widmann. In Id., Dialettica della razionalizzazione (pp. 221-264). Milano, Unicopli, 1983.

Habermas J. (1985). Trad. it. Il discorso filosofico della modernità. Bari-Roma: Laterza, 1987.

Habermas J. (1988). Trad. it. Intervista a Robert Maggiori. In Id., La rivoluzione in corso (pp. 32-40). Milano: Feltrinelli, 1990.

Habermas J. (1989). Intervista con Hans Peter Krüger. In Id., La rivoluzione in corso (pp. 86-102). Milano: Feltrinelli, 1990.

Habermas J. (1992). Fatti e norme. Contributi a una teoria discorsiva del diritto e della democrazia. Milano: Guerini e Associati, 1996.

Habermas J. (1999a). Trad. it. Filosofia ermeneutica e filosofia analitica. Due varietà complementari della svolta linguistica. In Id., Verità e giustificazione. Saggi filosofici (pp. 61-95). Bari-Roma, Laterza, 2001. 


\section{Corchia}

Habermas J. (1999b). Ancora una volta: sul rapporto tra teoria e prassi. In Id., Verità e giustificazione. Saggi filosofici (pp. 313-325). Bari-Roma: Laterza, 2001.

Habermas J. (2006). Il ruolo dell'intellettuale e la causa dell'Europa. In Id., Il ruolo dell'intellettuale e la causa dell'Europa (pp. 5-13). Roma-Bari: Laterza, 2011.

Held D. (1978), The Battle over Critical Theory. Sociology, 12, 3: 553-560.

Hviid Jacobsen M. (ed.) (2016). Beyond Bauman: Critical Engagements and Creative Excursions?. London: Routledge.

Hviid Jacobsen M., Poder P. (eds.) (2008). The Sociology of Zygmunt Bauman: Challenges and Critique. London: Ashgate.

Jedlowski P. (2013). La modernità in molte forme. In Th. Großbölting, M. Livi, C. Spagnolo (a cura di), L'avvio della società liquida? Il passaggio degli anni Settanta come tema per la storiografia tedesca e italiana (pp. 83-108). Bologna: il Mulino.

Jedlowski P. (2013). La modernità in molte forme. In Th. Großbölting, M. Livi, C. Spagnolo (a cura di), L'avvio della società liquida? Il passaggio degli anni Settanta come tema per la storiografia tedesca e italiana (pp. 83-108). Bologna: il Mulino.

Junge M. (2006). Zygmunt Bauman: Soziologie zwischen Moderne und Flüchtiger Moderne: Eine Einführung. Wiesbaden: VS Verlag für Sozialwissenschaften.

Kilminster R., Varcoe I. (eds.) (1995). Culture, Modernity and Revolution: Essays in Honour of Zygmunt Bauman. London: Routledge.

Leccardi C. (2008a). Zygmunt Bauman: sociologia critica e impegno etico nell'epoca della globalizzazione. In Ghisleni M., Privitera W. (a cura di). Sociologie Contemporanee. Bauman, Beck, Bourdieu, Giddens, Touraine (pp. 3-43). Novara: Utet Università.

Leccardi C. (2008b). Dentro l'individualizzazione. Etica della responsabilità e politica. In Bauman Z., Individualmente insieme (pp. 9-28). A cura di C. Leccardi. Reggio Emilia: Diabasis.

Magatti M. (2003). Zygmunt Bauman: un interprete del nostro tempo. In Bauman Z., Una nuova condizione umana (pp. 7-34). Milano: Vita e Pensiero.

McCarthy Th. (1978). The Critical Theory of Jurgen Habermas. Cambridge (MA): The MIT Press.

Müller-Doohm S. (2003). Theodor W. Adorno. Biografia di un intellettuale. Roma: Carocci, 2003

Müller-Doohm S. (2014). Jürgen Habermas. A Biographie. Berlin: Suhrkamp.

Müller-Doohm S. (2018). Negazione e argomentazione. La teoria critica di Adorno e Habermas. Torino: Nuova Trauben edizioni.

Palese E. (2012). La filosofia politica di Zygmunt Bauman: individuo, società, potere, etica, religione nella liquidità del nostro tempo. Milano: Mimesis.

Penati G. (1977). Cultura come prassi by Zygmunt Bauman. Rivista di Filosofia NeoScolastica, 69(3): 541-544.

Picchio M. (2012). I vicini lontani. Sociologia dello straniero attraverso Simmel, Bauman e Beck. In M.C. Federici, M. Picchio (a cura di), Pensare Georg Simmel: eredità e prospettive (pp. 341-460). Perugia: Morlacchi.

Pusey M. (1987). Jürgen Habermas. London: Routledge. 


\section{Corchia}

Rattansi A. (2017). Bauman and Contemporary Sociology: A Critical Analysis. Oxford: Oxford University Press.

Ray L. (2007). From postmodernity to liquid modernity. What's in a metaphor?. In Elliott A. (ed.), The Contemporary Bauman (pp. 63-80). London: Routledge.

Smith D. (1998). Zygmunt Bauman: How to Be a Successful Outsider. Theory, Culture and Society, 15, 1: 39-45.

Smith D. (1999). Zygmunt Bauman: Prophet of Postmodernity (Key Contemporary Thinkers). Cambridge: Polity.

Tester K. (2004). Trad. it. Il pensiero di Zygmunt Bauman. Trento, Erickson, 2005.

Tester K., Hviid Jacobsen M. (2005). Bauman Before Postmodernity: Invitation, Conversations and Annotated Bibliography 1953-1989. Aalborg: Aalborg University Press.

Tester K., Hviid Jacobsen M., Marshman S. (2007). Bauman Beyond Postmodernity: Conversations, Critiques and Annotated Bibliography 1989-2005. Aalborg: Aalborg University Press.

Therborn G. (2006). After dialectics: postmodernity, post-Marxism, and other posts and positions. In Gerard Delanty (ed.), Handbook of Contemporary European Social Theory (pp. 185-202). New York-London: Routledge.

Wolff K.H. (1983). Surrenderand-and-Catch and Critical Theory. Praxis International, 3, 2: $147-160$. 